\title{
A Train-the-Trainer Programme to Deliver High Quality Education for Healthcare Providers
}

\author{
Jessica L Peck
}

Please cite this article as: J L Peck, 'A Train-the-Trainer Programme to Deliver High Quality Education for Healthcare Providers', Anti-Trafficking Review, issue 17, 2021, pp. 140-147, https://doi.org/10.14197/atr.201221179

As a paediatric nurse practitioner, professor of nursing, and national officer of the oldest and first advanced practice nursing organisation in the world, I consider myself to be engaged and informed on matters of child health. In 2016, a friend who runs an anti-trafficking organisation asked me to help create a continuing education programme for nurses to raise awareness about human trafficking. Their organisation provided aftercare services and was alarmed at the first-hand reports of victims' healthcare encounters without any assessment for risk of victimisation. At the time, I declined, not considering myself to be an expert in the subject of trafficking. I planned to refer my friend to a nurse expert but was astonished when I could not identify anyone.

As I learnt about the significant connection between healthcare and human trafficking, I also became aware of the overwhelming silence of nursing responses in academic institutions, healthcare organisations, and professional societies. In the following months, I encountered two teenagers and one school-aged child in my clinical practice who were victims of trafficking. I came face-to-face with a survivor who was arrested in the hotel of our national professional nursing organisation's annual conference. As I encountered more children who had been exploited and victimised, I recognised the need to engage the nursing profession as a critical link in creating an evidence-informed health framework to respond to at-risk persons. 
As awareness of human trafficking in the United States increases, well-meaning but misguided training courses for healthcare providers also proliferate. ${ }^{1}$ This trend concerns me because most healthcare training courses I attended were presented by law enforcement, and a criminal justice paradigm is not linked to the healthcare environment. Many healthcare organisations I initially encountered refused education programmes on trafficking because of a perceived lack of applicability and the vicarious trauma reported by participants following a training.

In 2017, after recognising the need for a national-level nursing engagement in response to children victimised by trafficking, I helped lead the National Association of Pediatric Nurse Practitioners (NAPNAP) to establish Partners for Vulnerable Youth (a non-profit nursing organisation to serve selected populations of at-risk youth) and its first initiative: the Alliance for Children in Trafficking (ACT). I served as the founding chair, creating ground-level organisational support and infrastructure to sustain a national programme with the goals to improve nursing awareness of trafficking, equip nurses with evidence-based tools and resources, collaborate with multidisciplinary service providers, and engage as policy advocates to promote legislative and regulatory frameworks conducive to preventive healthcare efforts and victim-centred services.

With more than twenty-two partner organisations, ACT serves as a national leader coordinating and uniting efforts to equip nurses and other healthcare providers in effective anti-trafficking responses. ${ }^{2}$ A research survey of the NAPNAP membership $(n=8,647)$ revealed 99 per cent of respondents felt child trafficking was a critical paediatric health issue. Although 87 per cent believed it was possible that they might encounter a potential victim in their clinical practice, only 24 per cent reported confidence in their ability to identify an at-risk child. Additionally, while 91 per cent of respondents believed children were exploited sexually, only 78 per cent recognised labour trafficking as a form of abuse and exploitation. Less than one third felt comfortable discussing the long-term health needs of potential victims. Many nurses anecdotally reported that the lack of awareness, education, and organisational preparedness led to figuring out processes when a patient was actively in the care environment. Some nurses reported reticence to speak up for fear of the relationship of victimisation and behaviours designated

1 JD Houston-Kolnik, C Soibatian, and MM Shattell, 'Advocates' Experiences with Media and the Impact of Media on Human Trafficking Advocacy', Journal of Interpersonal Violence, vol. 35, issue 5-6, 2017, pp. 1108-1132, https://doi.org/10.1177/ 0886260517692337; S Litam and E Lam, 'Sex Trafficking Beliefs in Counselors: Establishing the need for human trafficking training in counselor education programs', International Journal for the Advancement of Counselling, vol. 43, 2020, pp. 1-18, https:// doi.org/10.1007/s10447-020-09408-8.

2 National Association of Pediatric Nurse Practitioners Partners for Vulnerable Youth, 'Alliance for Children in Trafficking', retrieved 18 November 2020, https://www. napnappartners.org/alliance-for-care-coordination. 
as criminal, with disturbing reports of patient arrest or deportation following such reports. This situation caused retraumatisation for the victims and vicarious trauma to the nurses.

In response, ACT developed a three-part continuing education series together with the American Academy of Pediatrics for dual continuing medical and nursing education. This training series, created in compliance with guidelines published by Health, Education, Advocacy, and Linkage (HEAL) Trafficking, has been disseminated to more than 1,000 providers. $^{3}$

I began engaging as the sole nursing representative with the National Human Trafficking Training and Technical Assistance Center (NHTTAC), a division of the Office of Trafficking in Persons at the United States Department of Health and Human Services, to help a multidisciplinary team create a technical report outlining core competencies for medical, nursing, social work, and behavioural health professionals. These competencies are a critical first step in standardising responses to trafficking across disciplines, while specifically addressing core competencies of individual clinicians, healthcare organisations, and academic institutions. The report's co-authors, including myself, are engaged in multiple media and healthcare professional outlets to raise awareness of this tool, which will help establish nomenclature and data-reporting standards by which to measure outcomes. ${ }^{4}$

\section{Train-the-Trainer Programme}

In 2018, we saw the need for a train-the-trainer programme (ACT Advocates) and solicited volunteers. Our first training programme drew more than 100 nursing attendees at a national conference. ACT Advocates training requires participants to take designated prerequisite courses including NAPNAP Partners' three-part (3-PARRT, Provider Assessing Risk and Responding to Trafficking) training course; a HEAL Trafficking course entitled Rethinking Representation: Framing Human Trafficking for Health Professionals; and NHTTAC's SOAR to Health and Wellness Training. After these courses are complete, trainees take a two-hour instructional course covering the policies and procedures governing participation in the ACT Advocates programme.

3 Ibid.

4 Office on Trafficking in Persons, An Office of the Administration for Children \& Families, 'Core Competencies for Anti-Trafficking Response in Healthcare Systems Release,' 24 February 2021, retrieved 5 April 2021, https://www.acf.hhs.gov/otip/ news/core-competencies-anti-trafficking-response-healthcare-systems. 
Careful consideration was given to an innovative pedagogical approach and curriculum design. The core training tenets are evidence-based, culturallyresponsive, patient-centred, trauma-informed, and survivor-advised. Trainees learn about trafficking through three primary lenses: 1) global overview of trafficking through an evidence-based framework, 2) trafficked persons in healthcare settings, and 3) responding to the needs of trafficked persons with multidisciplinary service providers, advocates, and law enforcement. Trafficked persons present to many kinds of care environments, including emergency care, primary care, specialty care, aesthetic or cosmetic care, and others. Presentation to care environments is preceded by an acute health complaint, which often precludes the victim from being able to perform forced acts of labour or sex. Nurses are highly skilled providers of care who can use clinical pathways and protocols to work within their scope of practice to identify risk and provide connections to appropriate services. Nurses recognise the health impacts of psychological and emotional trauma, and the intersection with physical health. I have seen nurses take training on human trafficking and leave devastated because they recognise potential victimisation of a past patient and feel moral distress at not having recognised it or responded appropriately. Given the complex intersection of criminality and healthcare presentations of trafficked persons, nurses can feel moral distress about knowing what to report, how to report, and to whom to report without fear of personal criminal backlash or unintentional harm to patients in the absence of trauma-informed care.

To respond to this professional situation, ACT Advocate trainees are required to take a course on trauma-informed care and its integration into healthcare settings for persons who are trafficked or at risk of trafficking. Trainees are instructed to give culturally-responsive and ethical representation of victims and survivors. As noted in the Core Competencies, a culturally-responsive approach supports interactions with patients based on culturally-informed practices and adopts a posture of learning. Culture spans beliefs, practices, and social formations of groups based on ethnicity, race, socioeconomic status, gender, sexuality, religion, ability, language, and any other social identity deemed significant to the patient. Ethical representation centres around avoiding voyeurism or the exploitation of victims and survivors, as well as condemning sensationalistic and biased imagery.

Trainees are provided with a NAPNAP Partners weblink to a curated collection of evidence-based resources, reviewed and collated by a diverse professional work group, and used as a guide for the development of organisational policies and protocols in healthcare settings. Currently, there are very few protocols and policies, leaving nurses and other clinicians to piece together clinical orders on their own in the absence of any organisational guidelines. Therefore, ACT provides nurses with protocol and policy resources and exemplars to respond to child trafficking in clinical settings. Online updates are posted with research articles, governmental resources, and other organisational tools and clinical supports, including exemplar protocols and organisational policies. Coalition building and 
interprofessional collaboration are key in protocol design. Emphasis is also placed on effectively combating common myths and misrepresentations that prevent adequate identification of risk. The training concludes with an expert speaker delivering a presentation with a slide deck they can use in future presentations, followed by a careful review of the policies and procedures to ensure scientific rigor and adherence to standardisation of presentations and advisement given.

After training, ACT Advocates are placed into a speaker's bureau and can be contacted by healthcare organisations or groups that want to learn more about human trafficking. ACT Advocates also serve as partner resources for clinical professionals and organisations striving to enact policies, procedures, and protocols. On the state level, ACT provided support for the successful passage of House Bill 2059, which requires direct care providers in Texas to take one hour of mandated continuing education on human trafficking. ${ }^{5}$ The curriculum went through peer review with survivor input and underwent anonymous evaluation by a third-party expert entity (HEAL Trafficking), which developed an evidencebased education checklist for auditing such programmes. Currently, surveys are being undertaken to measure the experience of the ACT Advocates, and the impact on skills, expertise, and self-efficacy. In a period of two years, ACT Advocates in more than 23 US states had reached audiences of nearly 20,000 nurses with nearly 1,000 more taking the advanced online training. ${ }^{6}$

There are now more than 100 ACT Advocates across the US. Post-programmatic surveys demonstrate respondents' increased feeling of self-efficacy in their ability to serve as a community experts and child trafficking advocates. Interest in ACT has grown over a two-year period, with nursing doctoral students using the programme as training and a certificate of endorsement, offering a platform for credibility in initiating scholarship, including theses and other evidence-based clinical initiatives. ACT Advocates maintain an online communication forum and have online meetings for sharing experiences and continuing to increase audiences and reach.

Commitments from ACT Advocates include the following: conduct at least two training sessions per year (no audience minimum); encourage organisations in their professional networks to develop policies, procedures, or protocols for healthcare encounters; complete post-evaluations; provide ongoing support to institutions and organisations; volunteer for organisational, academic, local, community, and state task forces; and assist in fundraising to continue this work. Presentation forums include nursing units, open conversation forums and learning arenas for

5 Texas Health and Human Services, 'Health Care Practitioner Human Trafficking Training', retrieved 18 November 2020, https://hhs.texas.gov/services/safety/texashuman-trafficking-resource-center/health-care-practitioner-human-traffickingtraining. 
healthcare providers and organisational trainings, healthcare organisation chapter meetings, professional conferences, and others. ACT Advocate trainings have resulted in 40 ACT Advocates in 35 states training more than 10,000 professionals nationally and internationally. This train-the-trainer approach has widened our ability to effectively reach clinicians with evidence-based training.

Required handouts for ACT Advocate presentations include ICD-10-CM coding for human trafficking from the American Hospital Association; the Joint Commission Advisory on Identifying Human Trafficking Victims in Healthcare; the trauma-informed approach to victim assistance in healthcare settings (PEARR tool) from Dignity Health; and the NAPNAP Partners resource page. The guidelines stipulate 50 minutes for presentations and 10 minutes for questions and answers. Participants agree not to modify the PowerPoint presentation except to insert local resources and statistics on one designated slide. ACT Advocates are also encouraged to partner with trafficking survivors to co-present.

During the COVID-19 pandemic, ACT Advocates adapted to a virtual educational environment, after in excess of 50 in-person presentations were cancelled. Healthcare attention is largely on COVID-19, which challenges our efforts to raise awareness of human trafficking. Virtual presentations have some advantages, including cost-effectiveness (no travel or meeting room fee), ease of attendance, improved connection to rural areas, compliance with physical distancing, and ease of marketing.

\section{Impacts of COVID-19 and the Need for Diversity, Equity, and Inclusion}

Recently, clarion calls across the nursing profession are prompting small but significant steps toward increasing diversity, equity, and inclusion. US nursing is quite a racially homogenous profession (80.8\% White; 6.2\% African American; 7.5\% Asian; 5.3\% Hispanic; 0.4\% American Indian/Alaskan Native; 0.5\% Native Hawaiian/Pacific Islander; $1.7 \%$ two or more races; and 2.9\% 'other'), which does not reflect the demographics of the American public. In response, the American Nurses Association, the National Black Nurses Association, the National Coalition of Ethnic Minority Nurse Association, and the National Association of Hispanic Nurses launched the National Commission to Address Racism in Nursing, tasked with the purpose of examining the issue of racism within the nursing profession and describing its impact on nurses, patients, communities, and healthcare systems. In addition, the National Academy of Medicine released a report entitled The Future of Nursing 2020-2030: Charting a Path to Achieve Health Equity. Within this report are specific recommendations to address social determinants of health and the provision of effective, efficient, equitable, and accessible care for all, and identifying and responding to system facilitators and barriers to promote a diverse nursing workforce. These actions emphasise the importance of accountability 
within the nursing profession to consider ways of teaching about trafficking and the associated risk factors without reinforcing power and privileges embedded in society. The ACT Advocate programme recognises the urgent need to support more racial diversity in the nursing workforce pipeline and NAPNAP has taken deliberate steps to do so. A Standing Committee on Diversity, Equity, and Inclusion launched in summer 2021 to take action to ensure that the organisational efforts and programmes are inclusive of diverse people and perspectives with equitable opportunities to participate and lead.

As with any new effort, the challenges have been significant. According to the Health Belief Model, persons considering actions to respond to health threats must have a perception of both susceptibility and severity along with realistic expectation of perceived benefits. Raising awareness of the susceptibility and severity of health systems encountering victims of child trafficking has been a sustained effort with slow momentum.

With the outbreak of COVID-19, the healthcare world has been focused on the pandemic response, slowing previous gains for anti-trafficking efforts. Rising tensions over racism and reports of police brutality create tremendous barriers for effective interprofessional paradigms between healthcare and law enforcement. There are widely varying city, county, and state laws governing US legal responses to trafficked persons, leaving nurses unprepared and worried about their professional licenses when working in different locations. Nurses are unsure about their legal responsibility to provide information or cooperate with law enforcement, or how to fulfil mandatory reporter obligations in the absence of organisational training, support, and policy norms. In some contexts, law enforcement is legally permitted to provide untrue information to victims as a means to elicit information in an investigation. Nurses are trained with emphasis on honesty and transparency with patients, preserving autonomy in decisionmaking whenever possible. Thus, these professions can be in opposition while both voicing a goal of the best possible victim outcomes. Nurses may be afraid to report trafficking, fearing unintentional criminal retribution or deportation of their patients. Often, law enforcement will be present with the patient in a care environment, creating opposing priorities between law enforcement emphasising a criminal investigation and healthcare providers emphasising physical and psychosocial health needs. A collaborative victim-centred approach is optimal, but it requires coalition building. I have been to anti-trafficking convenings where healthcare is excluded as a multidisciplinary partner and viewed as obstructive to efforts of law enforcement.

As health professions participate in anti-trafficking education initiatives, nursing is emerging as a leader for cross-sector collaboration and a public health preventive approach. By contrast, law enforcement uses a downstream approach, focused on addressing problems that already exist without directed efforts at mitigating causative factors. The public health, or upstream approach, embraced by nursing 
focuses on prevention, addressing social, economic, political, environmental, and health factors with emphasis on early intervention. These efforts would be most effective in collaborative concert, simultaneously implementing both approaches.

\section{Conclusion}

As a profession employing more than 3.8 million registered nurses in the US and 20.7 million out of 43.5 million total health workers worldwide, nursing is by far the largest healthcare workforce in the world. Skilled in therapeutic communication, and having longer encounters with patients, nurses are ideally situated to provide a skilled response when trafficking risks are identified.

Nurses already employ effective prevention programmes for many issues, including environmental health, climate change, the opioid crisis response, and responding to social determinants of health. They can also employ trafficking prevention and early identification strategies. In particular, advanced practice nurses are positioned to lead the profession in providing a trusted voice of education to the public. Equipping nurses with evidence-based knowledge and skills can amplify anti-trafficking advocacy, especially when nursing is already engaging in initiatives for diversity, equity, and inclusion, while raising awareness of implicit racial bias. NAPNAP Partners' train-the-trainer programme has succeeded in reaching healthcare providers and community leaders with evidence-based, patient-centred, trauma-informed, and culturally-responsive training to equip and engage individuals and institutions to respond to trafficking situations effectively.

Dr Jessica Peck DNP, APRN, CPNP-PC, CNE, CNL, FAANP, FAAN is an expert paediatric nurse practitioner and anti-trafficking advocate who develops and leads inclusive and diverse interprofessional teams to provide outcomes of high-quality healthcare. She served as founding chair of the Alliance for Children in Trafficking, a national campaign of the National Association of Pediatric Nurse Practitioners Partners for Vulnerable Youth, where she worked with other national organisations to equip healthcare professionals. Dr Peck obtained a doctorate in Nursing Practice from the University of Alabama and currently serves as Professor of Nursing at Baylor University in Dallas, Texas. Email: jessica_peck@baylor.edu 